\title{
Temporal Patterns of Subjective Experiences and Self-Regulation during Ramadan Fasting among Elite Archers: A Qualitative Analysis
}

\author{
* Corresponding Author; \\ Address: Sport Psychology Centre \\ National Sports Institute of Malaysia \\ Bukit Jalil, 57000 \\ Kuala Lumpur, Malaysia \\ E-mail: jollyroyisn@hotmail.com
}

Received: Aug 09, 2011

Accepted: Aug 27, 2011

Key Words: Self Regulation; Qualitative Research; Ramadan Fasting; Subjective Experiences
Jolly Roy*1, PhD; Shazarina Hamidan ${ }^{1}$, MSc; Rabindarjeet Singh ${ }^{2}$, PhD

\section{INTRODUCTION}

$\mathrm{A}^{\prime}$ rchery is a sport where physical and psychological components can have effect on competition outcomes. One of the factors that distinguish top archers from those who are moderately successful is the ability to self- regulate in adversity. One such context is sports training during Ramadan fasting. Typically, the calendar of international sporting competitions rarely considers the Ramadan fasting period. For instance, the London Olympics will fall right in the middle of the Ramadan month in 2012. Muslim athletes have engaged in Ramadan fasting from long ago. Therefore, Muslim athletes rarely refrain from training and competing during Ramadan. The adherence to socio-religious practices during this period, leads to disruptions in the normal routine, which requires athletes to self-regulate themselves 
across the four weeks of the fasting month.

Archery places a great deal of physical and psychological demands on the athlete to maintain consistency. In this study, however, the major emphasis is on the individual athlete's subjective experiences as a vital component for athletic training. Little attention has been given to explore the athletes' performance related subjective experiences with qualitative methods. Therefore, the purpose of this research was to address this shortcoming by qualitatively examining the individual's subjective experiences and contextualize these experiences within the framework of self-regulation during Ramadan. Self-regulatory beliefs and processes are related to one's self-generated thoughts, feelings, beliefs and actions for goal directed behavior ${ }^{[1,2]}$. Thus, our research questions explored the individual athlete's performance related subjective experiences across four weeks of fasting. More specifically, we investigated what experiences, thoughts, feelings and beliefs were involved during Ramadan while the athlete was training. A secondary aim was to take forward what has been learned through anecdotal evidence with elite athletes, and translate it into applied settings in preparation for major events such as Olympics or world championships.

We believe that an individual's subjective experiences are the result of the interaction among personal, behavioral and environmental processes. The key role, however, revolves around the individual's thoughts, attitudes and personal beliefs within the environment, (i.e. Ramadan fasting) influencing the behavioral outcome. Zimmerman's self-regulatory model is designed to explain the thoughts and beliefs within the learning context; however, his framework was used here to understand the thoughts and experiences leading to self- regulation across the four weeks of Ramadan fasting ${ }^{[2,3,4]}$. In the light of Zimmerman's self-regulation, the personal component has three phases: forethought, performance control, and self-reflection that occurs across a temporal frame ${ }^{[2,3,4]}$.

The forethought phase involves an individual's feelings, thoughts and beliefs before initiating a task (i.e. Ramadan fasting in this context). The forethought phase helps an individual to follow a behavioral action through self-motivational beliefs ${ }^{[3]}$. For example, the cognitions during Ramadan fasting involve planning for appropriate nutritional intake to stay hydrated and endure the physical and mental discomfort of fasting. An individual's self-efficacy ${ }^{[5]}$ and personal values related to self-motivational beliefs, orients the person toward achieving the goal (e.g. fasting and sports performance in this research context).

The second phase is the performance control phase ${ }^{[3]}$ where the individual attempts the actions. The components include self-control, focusing, selfinstructions, self-experimentation and strategies to improve the quality of the activities. In this research context, the self-control involves curbing the desires to eat food and take fluids as in usual days. Selfexperimenting comprises of adopting different training strategies such as adjustment of training load or changes in the time of training. The athlete is also constantly monitoring his/her personal thoughts and behavioral actions in the fasting environment. Selfinstruction, persuasion to continue fasting and sports training can also be considered as a component of control. Thus, the control phase in this context is adapted to the action of fasting.

The third phase comprises of self-reflection ${ }^{[4]}$ which relates to an individual's response and reaction after attempting an action (e.g. fasting). Self-judgment is another component of this phase where the individual evaluates his own actions. In this research context, the athlete evaluates his/her training and sports performance during fasting and attributes the accomplishments to his/her own beliefs, ability and efforts. The self-reactions and inferences of the outcome, form the basis of subjective experiences which provide an index for better adapting to the fasting conditions, across a temporal dimension. For example, if the athlete attributes the experiences in a positive frame, he/she would believe in maintaining the outcome. On the contrary, if the experiences were attributed negatively, an individual would strive to adopt a new path or strategy. In a sport context, where the demands of international competition are very high (e.g: London Olympics, during the fasting month), the individual may choose to adopt a protective strategy such as to refrain from fasting or postpone fasting dates to avoid feelings of dissatisfaction due to failure. Selfregulation and adaptations in a precision sport such as 
archery are of particular interest in the context of Ramadan fasting because the psychological demands are constantly in a flux, given the duration of fasting and the intensity of performance during high-level competition. At a practical level, adaptation is an end point in a process where individuals respond positively to hardship, challenges, and threat ${ }^{[6]}$. This implies that practitioners require greater insights into the intraindividual dynamics of experiences in order to better understand idiosyncratic organization of self-regulation across the temporal dimension of Ramadan context.

Many of the studies ${ }^{[7,8,9,10]}$ conducted during Ramadan have addressed physiological and nutritional aspects with minor emphasis on psychological aspects ${ }^{[11]}$ such as irritability, cognitive functions, and consequences of sleep deprivation. To date, few studies have focused on the athlete's subjective experiences during Ramadan fasting. Further, it was also indicated ${ }^{[6]}$ that the majority of the research address the the individual's effort to adaptation and not how the environment might also adapt to meet an athlete's needs. Thus, it was felt that a greater understanding of an athlete's experiences would make an important contribution to design appropriate interventions to cope effectively in applied settings. While Muslims in some countries are moderate in their approach to Ramadan fasting, it is observed strictly in certain countries. A major competition such as London Olympics 2012, fall during the Ramadan fasting month and this marks the relevance of the study.

\section{METHODS AND SUBJECTS}

\section{Participants:}

Eleven elite Malaysian Muslim archers (male $=9$, female $=2 ; \mathrm{M}_{\mathrm{age}}=20.1 \mathrm{y}, \mathrm{SD}=2.8$ ) volunteered for the study. Purposive sampling was used to ensure that participants had been fasting during Ramadan in the year 2010. Purposive sampling involves researchers targeting participants closely related to the topic of research. All athletes had competitive experiences at the national and international level. All athletes were training together as a squad during the period of investigation. The athletes were training a total of six hours a day (three hours in the morning and three in the afternoon).

\section{Procedure:}

The Institutional Research Committee granted approval and ethical permission for the study. We explained the study procedures (e.g. study topic, significance of the study, rationale, confidentiality of responses) prior to signing the informed written consent.

\section{Interview Guide:}

Interviews were conducted during the three weeks after

Ramadan with an open-ended questions on their subjective experiences across the fasting period laying emphasis on the first half and second half of the month. Although the interviews began in the first week after Ramadan, most athletes were away in their native places, and were available in the second and third week after Ramadan. The responses to the questions were tape recorded with the consent of the participants. We ensured that the participants had enough time to recollect their experiences during Ramadan. There was no specific time limit set to respond to the questions and participants were free to add in as many details as possible whenever they remembered any new experiences. The individual interviews were in the presence of the first two authors, however, to maintain uniformity the second author conducted all the interviews.

Each individual athlete was encouraged to recall the experiences during the fasting month. The athlete responded to the question "Could you describe your experiences (pertaining to training/competition) during the first two weeks and last two weeks of Ramadan fasting". Probing and follow up questions were used to elicit more elaborations from the athletes.

\section{Data analyses:}

Grounded theory was employed for this research. This approach involves letting themes emerge from the qualitative data. In the grounded theory approach it is common to use inductive logic. The components of categorization included origin, verification, and temporal pattern. Origination stems from the athlete's responses; researcher's theoretical concepts and 
thoughts, and available literature. Verification included two strategies which were identifying rational and connecting the data to the category label, and referencing to seek support from the research data source. Temporal pattern refers to using knowledge that we already have to form categories. All recorded statements were transcribed verbatim generating 11 pages of single spaced text. We read all the interview data to gain familiarity with the information provided. This enabled us to identify the common and unique features of the interview text. The idiographic profile of the subjective experiences across the four weeks fasting period was developed. The statements extracted enabled the data reduction to meaningful qualitative data. We did an open coding ${ }^{[12]}$ by selecting statements relating to the research question as 'meaning units' and each was assigned a code with the first number representing the athlete, followed by the number of the meaning statement. We further reread the qualitative data for axial coding ${ }^{[13]}$. Following this we independently looked for patterns, explanation and temporal assessment to relate the statements to more general codes. To obtain a clear and valid set of codes, we compared our individual results. Research meetings for external validation helped to discuss emerging themes and reach consensus among coding decisions. Finally, we contacted the athletes with the framework of the coding and asked each athlete to confirm, refute or elaborate on our interpretations. Following this, the research partners acted as "devils advocate" ${ }^{[14]}$ in questioning the coding, to obtain more consistency among the researchers. Using the formula suggested by Miles and Huberman ${ }^{[15]}$ the reliability check showed a score of $89 \%$.

\section{RESULTS}

The focus of this study was to examine the temporal patterning of subjective experiences and selfregulation in athletes who were engaged in the time leading up to completion of Ramadan fasting. The analysis of the raw data theme yielded different dimensions such as physical, mental, emotional, behavioral, and spiritual. In the first two weeks of the Ramadan fasting month, the raw data theme yielded 15 first order themes (physical=5, emotional=3, behavioral=1, mental=3 and spiritual=3). In the second half also the raw data theme yielded 15 first order themes (physical=4, emotional=5, behavioral=1, mental $=4$ and spiritual=1). Overall patterns across the four weeks of fasting (Fig. 1) revealed that in the first two weeks of fasting, an increased percentage of athletes reported experiences associated with physical factors, emotional factors, behavioral factors, and spiritual factors. The mental factor, however, showed a different pattern, with the athletes reporting more experiences in the third and the fourth week of fasting.

\section{Physical:}

The general theme included issues related to physical factors with five first order themes. Seventy three percent of archers reported a feeling of tiredness only in the in the first two weeks of fasting. Within this theme, athletes commonly experienced exhaustion, sleep and thirst across the four weeks fasting period. Athletes reported bodily symptoms such as feeling dizzy only in the first two weeks. However, athletes did not mention any bodily symptoms in the second half of the fasting period. Instead, concerns related to performance such as skill execution surfaced in the interview. For instance, athlete 9 expressed:

"When I am fasting, I experience difficulty;....it is difficult to perform well. It is difficult in the training also".

\section{Emotional:}

The emotional experiences revealed variations across the temporal dimension of the fasting period. Patterns of emotions in the first two weeks indicated anger among $36 \%$ of athletes, irritation among $27 \%$ of athletes and exhaustion among $18 \%$ of athletes. For example, athlete 3 stated:

"I feel very thirsty...It is hot here....very uncomfortable. Easily fatigued...and..ehh... I get annoyed when I make mistakes during training”.

In the third and the fourth week, the emotional dynamics depicted a mixed response such as control, 
1st \& 2nd week $\square 3$ rd \& 4th week

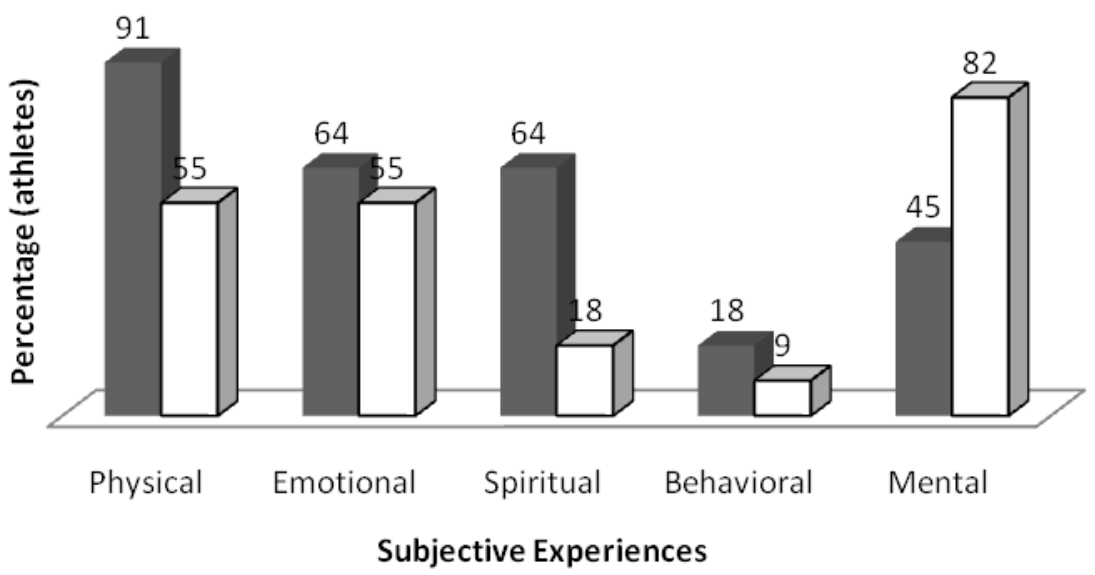

Fig. 1: Subjective experiences across Ramadan fasting

stress, excitement, lack of energy and desire to rest. For example, athlete 11 noted:

"I adjust to the situation..... and by this time...I am able to control my feelings and emotions. I also get excited.......you know...I feel excited as it is nearing the end of Ramadan fasting”.

\section{Behavioral:}

Athletes reported relatively lesser comments on behavioral aspects. In the first two weeks of fasting, the athletes experienced sluggishness. For example, athlete 9 stated:

"During Ramadan fasting, it feels like.... time is moving slowly....very slowly”.

In the third and fourth week, one athlete felt burdened. For example, athlete 4 stated:

"I feel very sleepy.... I have trouble getting up early... and then go for training...

I have to wake up....it is ok for the early meal...but I cannot miss the training, it is tedious”.

\section{Spiritual}

The higher order spiritual theme yielded gratification, unwavering and will power as the first order theme in the first two weeks of fasting. For example, athlete 6 noted:
"Well....I feel tired....but I have the will to fast".

In the third and fourth week, athletes reported better adaptation. For example, athlete 6 expressed:

"Well....during the second half I can perform better...... also got used to the fasting and training”.

\section{Mental:}

Forty five percent of athletes reported experiences related to mental factors in the first two weeks of the fasting. Inductive analyses revealed global dimensions such as personal experiences, liveliness, and focus. In the third and fourth week of fasting, however, 73\% of athletes reported experiences related to mental features such as distraction, lethargy, unwilling and habitual routines. Six athletes profoundly mentioned distraction as the most prominent mental factor affecting them. This Construct might best be described by the following quote from athlete 11:

“Archery needs a lot of concentration.....and we need to stay focused...We have long training hours... and one month of fasting!!.... ...it is difficult to stay focused when the body is also tired”. 


\section{DISCUSSION}

Our objective was to examine the temporal patterning of subjective experiences and self-regulation during the four weeks Ramadan fasting. The main dimensions that emerged from the interview were physical, mental, behavioral, spiritual, and emotional.

\section{Physical}

The qualitative results provided rich insight into the athlete's experiences and highlighted the numerous subjective experiences and adaptations between the first two weeks (initial phase) and the third and fourth week (latter phase). Although the themes were representations of idiosyncratic experiences ${ }^{[16]}$, some commonalities were evident. For example, exhaustion, sleep and thirst were a core group of experiences consistently reported by the athletes in the initial phase and latter phase of fasting. Previous studies have reported decreased sleep, daytime alertness and impaired psychomotor performance during fasting conditions $^{[8,10,17,18]}$. A closer examination of the raw data revealed that individual athletes probably engaged in a self-regulatory process across the fasting period. For instance, Athlete 2, 4, and 5 reported feeling exhausted in the initial phase of fasting. The same athletes, however, did not report feeling exhausted in the latter phase. This result is consistent with the findings that decreased psychomotor performances were observed in the first week of the fasting period ${ }^{[19]}$. It is possible that athletes' thoughts and beliefs ${ }^{[2,3,4]}$ concerning fasting are socio-culturally ingrained in the psyche which helped in monitoring personal and behavioral elements within the Ramadan context leading to efforts of self-regulation and better adaptation across the fasting period. It is also possible that the athletes use the social support and make a conscious effort to understand and deal with the contextual challenges of fasting, which is a core motive within the proposed adaptation framework ${ }^{[20] \text {. }}$

On similar lines, in the first phase of fasting, while $80 \%$ of athletes reported feeling tired, in the latter phase this theme did not emerge at all. Although athletes reported bodily symptoms such as feeling dizzy in the initial phase, in the latter phase, no mention of such experiences was made. Instead, matters related to skill executions emerged which suggests that the athletes were able to self- monitor and self-evaluate the required regulations in the context of Ramadan fasting. These findings also provide an indication of how an athlete perceives the need for adapting to the changing situations in the Ramadan context. Thus, despite hardships, athletes make all attempts to boost persistence across the fasting period.

\section{Emotional}

Considerable inter-individual differences existed in the emotion regulation. In the initial phase of the fasting month, the archers experienced anger, irritation and feelings of giving up. Studies have also reported a decrease in mood rating during Ramadan fasting ${ }^{[19]}$. In the latter phase, however, athletes reported better control, consistent with the performance control phase suggested by Zimmerman, indicating a possible adaptation of the body's metabolic system and a conscious effort taken by the athlete to self-regulate ${ }^{[3]}$. Evidence for this can be drawn from the statement "I got adjusted to the situation", which also reflects on the processes of self-reflection and self-judgment ${ }^{[3]}$. The other reported subjective experiences such as adaptation to pressure and effort to maintain liveliness also suggest how athletes strive to regulate across time. One plausible explanation is that, over the years, the athletes would have observed the regulatory effort in other athletes and learned to emulate the self-control, as a process of self-regulation. Self-regulation and adaptation would have stemmed from the understanding of self within the Ramadan context. Self-reactions ${ }^{[4]}$ involve an individual's satisfaction level with the outcome and subsequent inferences of a task. These inferences provide the raw material for individual perception to adapt to the required changes for enhanced self-regulation. More specifically, we believe that athletes might have taken self-regulatory strategies from the social context and transferred them to individual context. This can also be explained using the self- efficacy theory ${ }^{[5]}$ where vicarious experiences are a major source of self-efficacy. It can be elaborated within the concept of participatory modeling ${ }^{[5]}$ where the athlete learned about dealing with Ramadan fasting and sports training, firstly from the seniors in the team and secondly from the fasting individuals of the 
society. Schinke et al ${ }^{[6]}$ observed that a majority of the research about sport adaptation pertains to how an individual adapts and not how the environment might adapt to meet the individual's need ${ }^{[6]}$. It would be interesting therefore to mention that the usual practice of closing most of the restaurants and food courts in some Muslim dominated countries during the fasting period of Ramadan (day time), is a pathway serving as contextual adaptation to facilitate self-control.

\section{Behavioral}

The athletes experienced sluggishness in the first two weeks of fasting. They elaborated this experience as the "time moving slowly" during the fasting period. In support of this, Wilson ${ }^{[21]}$ observed that disruptions in sleep patterns, partial reversal of usual eating and fluid intake patterns and prolonged diurnal fast have a distinct impact on human circadian rhythms. These effects manifest in different ways, depending on the sunrise and sunset of the geographical location, weather conditions and socio-cultural practices.

Beside the sluggish feelings, the athletes also reported "feeling uncomfortable". In the third and fourth week of fasting, an athlete reported difficulty to wake up, which we coded as burdensome. Mental fatigue, especially sleepiness can have the greatest impact on athletes. It was found that sleep deprivation caused subjects to have slower reaction times and tend to miss stimuli over a test period of two hours ${ }^{[22]}$.One athlete reported tedious feelings. Although small in number, we cannot grossly ignore this, as 'tedious' feelings can interfere with the performance of archery. This finding provides a potential avenue for future investigation.

\section{Mental}

One of the significant findings is that $45 \%$ of athletes reported individual experiences, liveliness, and a shift in focus in the first two weeks of fasting. In support of this experience, a decrease in memory and work performance was demonstrated in studies involving university students ${ }^{[17,23]}$. Generally, it is expected that athletes would be better adjusted towards the latter part of the fasting month. Contrary to this expectation, this study revealed that an increased percentage of athletes reported experiencing distraction, lethargy, and unwillingness in the latter half of the fasting month. We do not wish to overstate the importance of sleep and diet among athletes, nevertheless, studies have reported impaired human performance due to sleep disturbance ${ }^{[24]}$, hypo hydration ${ }^{[25]}$ and energy deficit ${ }^{[26]}$. It is not clear at this point if the athletes adopted appropriate strategies for self-regulation or if the strategies failed to produce the desired result.

\section{Spiritual}

Another finding of importance was that $63 \%$ of athletes reported spiritual elements as subjective experiences. Comments such as "I take pleasure in fasting" expressed gratification; "I am able to endure" expressed unwavering feelings. These comments are indicative to the findings that religious and contemplative backgrounds play important roles [27] .We coded expressions of determination as will power. The will to achieve the goal of sports training during fasting allowed the athletes to persist in continuing the task regardless of physical and psychological constraint. This can be explained within the selfreflection phase ${ }^{[4]}$ relating to an individual's response and reaction after an action (e.g fasting). In addition, through self-judgment ${ }^{[4]}$ the athlete might be evaluating his/her sports performance during fasting and attributes the accomplishments to personal beliefs, ability and efforts.

Muslims pay more attention to religious tasks in the holy month of Ramadan resulting in a positive psychological and physical status ${ }^{[28]}$. In plain terms, we can assume that self-regulation to control the habitual routines and counter the powerful desires, requires some kind of inner mechanism, which is most likely rooted in the augmented beliefs of tolerance and sacrifice during Ramadan. The spiritual dimension probably acts as a unifying force within the athletes, to evolve a common bond between the individual and perceptions of faith ${ }^{[29]}$, personal fulfillment ${ }^{[30]}$ and a strong personal value system ${ }^{[31]}$. Not all athletes report discomfort. Some athletes have stated pleasurable experiences of fasting. Evidence was found in studies ${ }^{[32]}$ which had demonstrated how motivational incentives (e.g. to be participating in Olympics) could offset depletion effects of fasting. Insofar, fasting may render shortage of fuel for normal functioning, but 
not necessarily impair the desire (motivation) to perform ${ }^{[33]}$. Thus, it is reasonable that some athletes experienced pleasure in fasting.

\section{Applied Implications}

From the applied perspective, the study provided a relatively clear and concise overview of subjective experiences during the Ramadan fasting month. Additionally, the study enlists information concerning the temporal patterns of self-regulation and experiences such as individual's attitudes, beliefs and preferences

of experiences in relation to fasting practices and sport training. Although most Muslim athletes are attuned to Ramadan fasting practices, a framework to prepare action plans to deal with situational demands is desirable. This study provides some practical implications for coaches and athletes. Given the variability in the temporal fluctuation of individual experiences and the nature of self-regulation, practitioners should engage in constructive discussions with the coaches and athletes to consider to modify training loads during Ramadan fasting. The focus should be on the appropriate quantity without compromising the quality of training.

As part of preparing athletes for training/ competition during the fasting month, athletes should be encouraged to consume an additional amount of fluid and food during the permissible period. To augment the intake of fluid, the athletes should consume their favorite foods and beverages of different flavors. This would ensure a somewhat overcompensation and euhydration state in the fasting athletes prior to the start of the day's fast. In addition, this would help prevent the fasting athletes from comparing with non fasting athletes and undermining self-confidence.

Catering to sport specific demands is important in any applied settings. Archery demands many mental energies and recovery is vital. Extended rest has been a preventive coping strategy that can help in selfregulation among the athletes during the fasting month ${ }^{[34]}$. Therefore, it would be ideal to advise athletes to minimize social activities and make deliberate effort to sleep early. Recognizing the significance of energy conservation during the fasting month, continuous education to minimize non-essential physical activities should be encouraged. Self-control was one of the proactive strategies used by the athletes ${ }^{[34]}$. Thus, practitioners in association with coaches could help athletes in developing and maintaining the skills of calmness. Additionally, athletes should develop greater willpower and determination for perseverance, which are akin to enduring the 'lack of food and fluid'. Finally, practitioners can utilize the suggestion that the athletes participating in high level competition during Ramadan should consider regulation strategies to suit individual needs ${ }^{[34]}$. These could include associating with fasting athletes to provide social support, conscious disengagement from thoughts of fasting and associated experiences and emotion regulation by generating positive association to the spiritual elements of Ramadan fasting. At a more practical level, it would be desirable to provide evidence of training/ performance progress (e.g performance profiling/ outcomes) to facilitate enhanced feelings of mental preparation during Ramadan fasting. Coaches should also consider structuring virtual training environments (e.g. imagery to convey feelings of calmness and will power) which provide opportunities for persistence in training. Since there are differences in the fasting regimen, cultural and social customs and behaviors between Muslims in different continents ${ }^{[35]}$, it would not be appropriate to generalize the results.

\section{Limitation}

There are several limitations of the present study. Due to the he retrospective nature of the study, it was not possible to fully consider absolute subjective experiences. The study relied heavily on the accuracy of the athlete's subjective recalls. A second limitation is that the study outcome is embedded within the sociocultural context of Muslim Archers living within Malaysia. Finally, the results do not offer any explanations into the mechanisms of self-regulatory processes.

\section{CONCLUSION}

This study has offered an insight into the subjective experiences of archers related to sport training 
/participation during Ramadan fasting. The theoretical perspective of subjective experiences during Ramadan indicated five major domains: physical, emotional, behavioral, mental and spiritual. Overall, the trend showed changes in the patterns of experiences among the major domains across the temporal dimension. Athletes reported increased subjective experiences in mental factors toward the latter half of the fasting period. Given that the athletes reported a variety of subjective experiences and self-regulatory processes occurring when engaged in Ramadan fasting, it would be appropriate to consider modifications in training and target intervention to suit individual needs. Practitioners should emphasize mental components of training, as these appear to be salient in archery performance. The theoretical perspective of different dimensions of experiences provide a plausible basis for continued research with other athletes from different countries.

\section{ACKNOWLEDGMENTS}

The research was supported by grants to Nazirah Gulam Mohamed from Universiti Sains Malayasia (304/CIPPT/6310083).

\section{REFERENCES}

1. Zimmerman BJ. Investigating self-regulation and motivation: Historical background, methodological developments and future perspectives. Amer Edu Res J 2008;45:166-83.

2. Zimmerman BJ. A social cognitive view of self-regulated academic learning. J Edu Psy 1989;81:329-9.

3. Zimmerman BJ. Sociocultural influence and student' development of academic self-regulation: A social cognitive perspective. In D.M. McIerney\& S.Van Etten (Eds). Big theories revisited Greenwich, CT: Information Age Publishing. 2004; Pp:139-64.

4. Zimmerman BJ. Attaining Self-regulation: A social cognitive perspective. In M.Boekaerts,P., R. Pintrich\& M.Zeidner (Eds). Handbook of Self-Regulation San diego, California: Academic Press. 2000; Pp:13-39.

5. $\quad$ Bandura AJ. Self-efficacy:The exercise of control New York: W.H. Freeman. 1997.

6. Schinke RJ, Tenenbaum G, Lidor R, Battochio RC. Adaptation in Action: The transition from research to intervention. Sport Psy 2010;24:542-57.

7. Aziz AR, Wahid MF, Png W, Jesuvadian CV. 2010,Effects of Ramadan fasting on 60 min of endurance running performance in moderately trained men. B J Sp Medi 2010;44:516-21.

8. Aziz AR, Png W. Practical tips to exercise training during the Ramadan fasting month. ISN Bull 2008;1:13-9.

9. Meckel Y, Ismaeel A, Eliakim A. The effect of the Ramadan fast on physical performance and dietary habits in adolescent soccer players. Eur J Appl Physiol 2008;102:651-7.

10. Roky R, Iraki L, HajKhlifa R, et al .Day time alertness, mood, psychomotor performances, and oral temperature during Ramadan intermittent fasting. Ann Nutr Meta 2000;44:101-7.

11. Kadri N, Tilane A, El Batal M, et al. Irritability during the month of Ramadan. Psychosomatic Med 2000;62:280-5.

12. Gratton C, Jones I. Research methods for sport studies. New York: Roultedge Taylor and Francis. 2004, Pp:220-221

13. Strauss AL, Corbin JA. Basics of qualitative research: grounded theory procedures and techniques. In G.D.Shank Qualitative Research (2 ${ }^{\text {nd }}$ ed). New Jersey: Pearson Merrill Prentice Hall. 2006 Pp: 130-1.

14. Krane V, Anderson M, Stean W. Issues of qualitative research methods and presentation. J Sport Ex Psy 1997;19:213-8.

15. Miles M, Huberman A. Qualitative Data Analysis. Thousand Oaks: Sage. 1994. 
16. Hanin YL. Emotions in Sport. Human Kinetics Champaign, IL. USA .2000.

17. Ali MR, Amir T. Effects of fasting on visual flicker fusion. Percept Mot Skills 1989;69:627-31.

18. Waterhouse J ,Atkinson G, Edwards B, Reilly T. The role of a short post-lunch nap in improving cognitive, motor, and sprint performance in participants with partial sleep deprivation. J Sport Sci 2007;25:1557-66.

19. Roky R, Houti I, Moussamih S, et al. Physiological and chronobiological changes during Ramadan intermittent fasting. Ann Nutr Meta 2004:48:296-303.

20. Fiske S T. Social beings: A core motives approach to social psychology. Danvers, MA: Wiley \& Sons. 2004

21. Wilson D, Drust B, Reilly T. Is diurnal lifestyle altered during Ramadan in professional Muslim athletes? Bio Rhy Res 2009;40:385-97.

22. Van den Berg J, Needy G. Performance on a simple reaction time task while sleep deprived. Percep Motor Skills 2006;102: 589-99.

23. Ennigrou S, Zenaidi M, Ben SlamaF, et al Ramadan and customs of life: Investigation with 84 adult residents in the districts of Tunis. La Tunise Médicale 2001;79:508-14.

24. Reilly T, Edwards B. Altered sleep-wake cycles and physical performance in athletes. Physi Beha 2007;90:274-284.

25. Malik GM, Mubarik M, Hussain T. Ramadan fasting effects on health and disease. J Assc Physi Ind 1996;44:332-4.

26. Rico-Sanz J, Zehnder M, Buchli R. Muscle glycogen degradation during simulation of a fatiguing soccer match in elite soccer players examined non-invasively by 13C-MRS. Med Sci Sport Exer 1999;31:1587-93.

27. Pargament KI, Ensing DS, Falgout K, et al. God help me: (I): Religious coping effort as predictors of the outcomes to significant negative life events. Am J Community Psychol 1990;18:793-824.

28. Akuchekian S, EbrahimiA, AlvandianS. Effect of the holy month of Ramadan on coping strategies. $J$ Res Med Sci 2004;2: 65-8.

29. Banks R Health and spiritual dimension: Relationships and implications for professional preparation programs. J Scho Health 1980;18:79-90.

30. Pilch JJ. Wellness spirituality. Health Values 1988;12:28-31.

31. Seaward BL. Spiritual well-being: A health education model. J Health Edu 1991;22:166-9.

32. Muraven M, Slessareva E. Mechanisim of Self-control failure: Motivation and limited resources. Pers Soc Psy Bull 2003;2: 894-906.

33. Galliot MT, Baumeister RF, DeWall CN, et al. Self-control relies on glucose as a limited energy source: will power is more than metaphor. J Per Soc Psy 2007;92:325-36.

34. Roy J, Hwa OC, Singh R, et al. Self-generated coping strategies among Muslim athletes during Ramadan fasting. J Sports Sci Med 2011;10:137-44.

35. Waterhouse J, Alkib L, Reilly T. Effects of Ramadan upon fluid and food intake, fatigue, and physical, mental, and social activities: a comparison between the UK and Libya. Chronobiol Int 2008;25:697-724. 